Sharif University of Technology
Scientia Iranica
SCIENTIA

\title{
A global Ritz formulation for the free vibration analysis of hybrid metal-composite thick trapezoidal plates
}

\author{
S. Shokrollahi and S. Shafaghat* \\ Department of Aerospace Engineering, Malek Ashtar University of Technology, Tehran, Iran.
}

Received 29 August 2014; received in revised form 3 January 2015; accepted 21 February 2015

\section{KEYWORDS}

Ritz method;

Hybrid plate;

Thick trapezoidal

plates;

Natural frequency.

\begin{abstract}
A general variational formulation for free vibration analysis of hybrid (metalcomposite) plates with a trapezoidal platform is presented in this paper. The plate is composed of two distinguished parts in the span direction, where the inboard section is assumed to be made of an isotropic metal, and the outboard section is from a laminated composite material. Kinematics, corresponding to the First-order Shear Deformation plate Theory (FSDT), is used to take into account the non-classic effects of transverse shear deformation and rotational inertia for both sections in the analysis. The developed approach is based on the global Ritz method, where the transverse deflection and two rotations of the plate are independently approximated by simple and Legendre polynomials. The algorithm allows one to obtain an approximate analytical solution for the hybrid plate with different geometric aspect ratios, numbers of layers, staking sequences and metal to composite ratios. The results of the present work for special cases, including pure metal and pure composite plates, are in good agreement with previous works.

(C) 2016 Sharif University of Technology. All rights reserved.
\end{abstract}

\section{Introduction}

For many years, mathematical models of aircraft wings based on equivalent plate representation combined with global Ritz analysis techniques have been used for basic studies in vibration, aeroelasticity and structural optimization [1-3]. These investigations were firstly based on isotropic metal plates. However, the current trend in aircraft design is to increase the proportion of fiber composites in the structures. During the last three decades, the use of advanced light weight-high strength Fiber Reinforced Polymer (FRP) composite materials in modern aircraft structures has rapidly increased [4]. For instance, about 20\% of the JAS39 Gripen structure is made of FRP composite material (Figure 1). Due to this revolutionary tendency in the aircraft industry towards the use of composite materials, many investigators have paid attention to developing approaches

\footnotetext{
*. Corresponding author. Tel.: +98911 7133627;

E-mail address: salmanshafaghat@mut.ac.ir (S. Shafaghat)
}

for the analysis of anisotropic laminated plates [5]. On the other hand, since many primary aircraft structural parts are also constructed using metals, the number of hybrid metal-composite structures is increasing. Based on the inherent property differences between composites metals [6], other certification and design methods adapted for the composites have had to be developed. It should be noted that most developed approaches for the vibration analysis of laminated composite plates with trapezoidal and triangular shapes are based on the theory of thin plates.

Classical Laminated Plate Theory (CLPT) neglects the effects of shear deformation and rotational inertia, and this leads to results that overestimate the frequencies of vibration. This error is greater when the thickness of the plate increases. The simplest alternative, considering the above mentioned effects, is the use of the first order shear deformation theory for moderately thick plates, proposed by Reissner and Mindlin, which incorporates the approximation made, with respect to non-uniform distribution strains and 


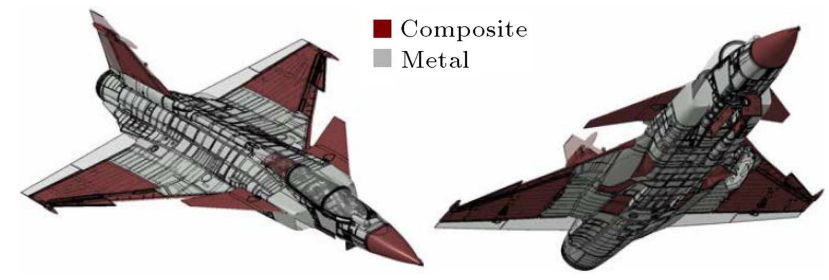

Figure 1. Composite and metal materials in JAS39 Gripen [4].

shear stresses. A complete analysis of the theoretical basis and the main advantages, application areas and the limitations of using FSDT or CLPT theories can be found (e.g. in [7]). Liew et al. [8] present a review of work on the vibration of thick plates, which mainly uses the first-order theory and refer to rectangular plates.

Particularly, the study of moderately thick trapezoidal plates through approximate analytical methods presents the difficulty of the construction of simple and adequate approximation functions that can be applied to the entire domain of the plate [9]. The global pb-2 Rayleigh-Ritz method was used to study the free vibration behavior of trapezoidal Mindlin plates [10] and cantilever triangular Mindlin plates [11]. Zhong [12] analyzed the free vibration of triangular plates by the differential quadrature method. Dozio and Carrera [13] proposed a variable kinematic Ritz formulation for vibration study of arbitrary quadrilateral thick plates. All this research work refers to plates made of isotropic material.

A few studies can be found in the literature for the free vibration analysis of laminated thick trapezoidal plates. For instance, the Rayleigh-Ritz procedure has been applied by Kapania and Lovejoy [14] in the analysis of quadrilateral, thick, generally laminated plates having arbitrary edge supports, together with Chebychev polynomials as trial functions. The boundary conditions have been enforced by the appropriate use of distributed linear and rotational springs along the edges. This method has been applied to cantilever plates. Chen et al. [15] studied the free vibration of cantilevered symmetrically laminated thick trapezoidal plates using p-Ritz method, incorporating the third-order shear deformation theory. Haldar and Manna [16] proposed a high precision triangular element with shear strain for the free vibration analysis of composite trapezoidal plates. Gurses et al. [17] used the method of Discrete Singular Convolution (DSC) for free vibration analysis of laminated trapezoidal plates. Gurses, et al. [17] obtained the governing equations and boundary conditions for the free vibration of trapezoidal plates using the First order Shear Deformation Theory (FSDT), together with proper transformation from a Cartesian system into trapezoidal coordinates; the Generalized Differential Quadrature (GDQ) method is then employed to obtain solutions. The vibration of Mindlin plates on elastic edge supports was analyzed in [18-20]. Maria et al. [21] presented a general vibrational formulation for the determination of natural frequencies and mode shapes of the free vibration of symmetric laminated plates of trapezoidal and triangular shape. Shokrollahi and Bakhtiari-Nejad [22] studied the effect of the sweep angle and taper ratio of swept back trapezoidal wings based on the geometrically nonlinear von Karman plate theory. Zamani et al. [23] studied the free vibration analysis of moderately thick symmetrically laminated general trapezoidal plates with various combinations of boundary condition. They employed the Generalized Differential Quadrature (GDQ) method to obtain solutions for the governing equations. They used the proper transformation from the Cartesian system into trapezoidal coordinates. They showed that this method has good accuracy and a fast rate of convergence. Civalek [24] analyzed the Discrete Singular Convolution (DSC) method that is developed for the vibration analysis of moderately thick symmetrically laminated composite square plates, based on the First-order Shear Deformation Theory (FSDT). In this approach, the derivatives in both the governing equations and the boundary conditions are discretized by the method of DSC. Wang and Wu [25] presented the free vibration analysis of rhombic plates with free edges using a modified Differential Quadrature Method (DQM). Due to the stress singularity at the obtuse angles of the rhombic plates, many approximate and numerical methods have encountered serious convergence problems when the skew angle is large. This problem is solved by DQM. Civalek [26] developed a four-node Discrete Singular Convolution (DSC) method for free vibration analysis of arbitrary straight-sided quadrilateral plates. He uses geometric transformation to transfer the governing equations and boundary conditions of the plate from the physical domain into a square computational domain.

In all these papers, the plates under consideration have either a fully metal or composite construct. The main objective of the present paper is to propose a general algorithm that allows obtaining approximate analytical solutions in order to study the free vibration of moderately thick trapezoidal hybrid plates. The procedure is based on the general Ritz method. Meanwhile, the interface connection between the metal and the composite parts is assumed to be continuous.

\section{Theoretical formulation plate geometry}

Consider a general trapezoidal hybrid plate with uniform thickness, $h$, and an arbitrary classical boundary condition. The Cartesian coordinate system, $x-y$, passing through the mid-surface of the plate, and the geometry of the plate with side lengths, $a, b$, 

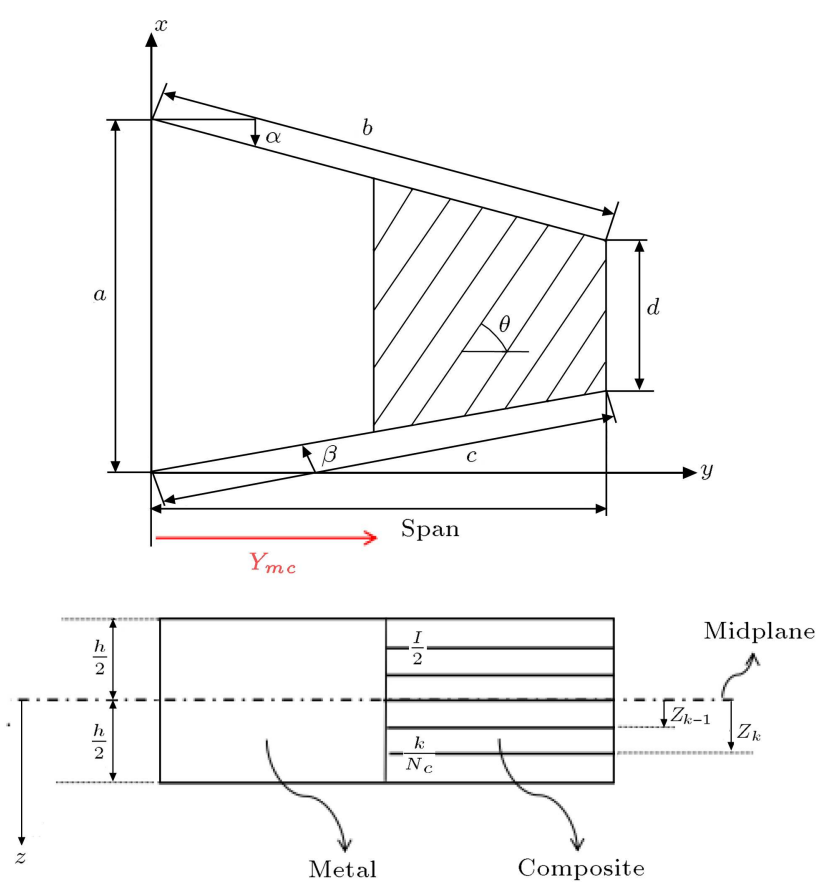

Figure 2. Geometry and notations of a hybrid plate.

$c, d$, and internal angles, $\alpha$ and $\beta$, are shown in Figure 2. The lamination scheme is symmetric with respect to the mid-plane for the composite part. The angle of the fiber orientation is denoted by $\theta$, which is measured from the $x$-axis to the fiber direction, as shown in Figure 2. Throughout the remainder of the paper, a counterclockwise, four-letter, compact symbolic notation is used for describing the plate boundary conditions. Letters $S, F$ and $C$ have been introduced to denote, respectively, simply supported, free and clamped edges.

The present study is based on the First order Shear Deformation plate Theory (FSDT). The components of the displacements field in the $x, y$ and $z$ directions, at any time, $t$, are given by:

$$
\begin{aligned}
& u(x, y, z, t)=z \theta_{x}(x, y, t), \\
& v(x, y, z, t)=z \theta_{y}(x, y, t), \\
& w(x, y, z, t)=w_{0}(x, y, t),
\end{aligned}
$$

where $w_{0}(x, y, t)$ are the deflections of the midplane points, and $\theta_{x}(x, y, t)$ and $\theta_{y}(x, y, t)$ are the rotations of the cross sections with respect to coordinates $x$ and $y$, respectively. For free vibration analysis, the displacement and rotations are given by harmonic functions of the time, i.e:

$$
\begin{aligned}
& w(x, y, t)=w(x, y) \cos \omega t, \\
& \theta_{x}(x, y, t)=\theta_{x}(x, y) \cos \omega t, \\
& \theta_{y}(x, y, t)=\theta_{y}(x, y) \cos \omega t,
\end{aligned}
$$

where $\omega$ is the frequency of the plate in radians per second.

According to Eqs. (2) to (4), the maximum kinetic energy of the freely vibrating plate expressed in the Cartesian coordinate is given by:

$$
T_{\max }=T_{\text {max }_{\text {metal }}}+T_{\text {max }_{\text {composite }}}
$$

where $T_{\text {max }_{\text {metal }}}$ is kinetic energy of the metal part, and $T_{\text {max }_{\text {composite }}}$ is the kinetic energy of the composite part. $T_{\text {max }_{\text {metal }}}$, in the Cartesian coordinates, are given by:

$$
\begin{aligned}
& T_{\max _{\text {metal }}} \\
& \quad=\frac{\rho_{m} h \omega^{2}}{2} \int_{0}^{Y_{m c}} \int_{\frac{(a-y)}{\tan \alpha}}^{\tan \beta}\left\{(w)^{2}+\frac{h^{2}}{12}\left(\theta_{x}^{2}+\theta_{y}^{2}\right)\right\} d x d y,
\end{aligned}
$$

where $\rho_{m}$ is the mass density of the plate metal part, $\omega$ is the circular frequency where the integration is carried out over the metal part of the plate, and $Y_{m c}$ is the length of the boundary between the metal and the composite.

Also, $T_{\text {max }_{\text {composite }}}$ in the Cartesian coordinates are given by:

$$
\begin{aligned}
& T_{\max _{\text {composite }}} \\
& \quad=\frac{\omega^{2}}{2} \int_{Y_{m c}}^{\operatorname{span}} \int_{\frac{y}{\tan \beta}}^{\frac{(a-y)}{\tan \alpha}}\left\{m_{c} h w^{2}+I_{c}\left(\theta_{X}^{2}+\theta_{Y}^{2}\right)\right\} d x d y,
\end{aligned}
$$

where $m_{c}$ and $I_{c}$ are the mass and moment of inertia of the composite, which are given in the following equations:

$$
\begin{gathered}
m_{c}=\int_{-\frac{h}{2}}^{\frac{h}{2}} \rho_{c} d z=\sum_{k=1}^{N_{c}} \rho_{c_{k}}\left(h_{k+1}-h_{k}\right), \\
I_{c}=\int_{-\frac{h}{2}}^{\frac{h}{2}} \rho_{c} d z=\sum_{k=1}^{N_{c}} \rho_{c_{k}}\left(h_{k+1}^{3}-h_{k}^{3}\right),
\end{gathered}
$$

where $\rho_{c}$ is the composite mass density. The maximum strain energy of the mechanical system is given by:

$$
U_{\text {max }}=U_{\text {max }_{\text {metal }}}+U_{\text {max }_{\text {composite }}},
$$

where $U_{\text {max }_{\text {metal }}}$ and $U_{\text {max }_{\text {composite }}}$ are the maximum strain energies of the metal part and composite part of the plate, respectively, and which, in the Cartesian coordinates, are given as:

$$
U_{\max _{\text {metal }}}=\frac{1}{2} \int_{0}^{Y_{m c}} \int_{\frac{(a-y)}{\tan \beta}}^{\tan \alpha} D\left(\left(\left(\frac{\partial \theta_{x}}{\partial x}\right)^{2}+\left(\frac{\partial \theta_{y}}{\partial y}\right)^{2}\right)\right.
$$




$$
\begin{aligned}
& \left.+2 v \frac{\partial \theta_{x}}{\partial x} \frac{\partial \theta_{y}}{\partial y}\right)+\frac{D(1-v)}{2}\left(\frac{\partial \theta_{x}}{\partial y}+\frac{\partial \theta_{y}}{\partial x}\right)^{2} \\
& \left.+k G h\left(\left(\theta_{x}+\frac{\partial w}{\partial x}\right)^{2}+\left(\theta_{y}+\frac{\partial w}{\partial y}\right)^{2}\right)\right\} d x d y
\end{aligned}
$$

In these equations, $k, G$ and $v$ are the shear coefficient, the shear modulus and Poisson's ratio for metal, respectively; $D$ is plate flexural rigidity, given by:

$$
D=\frac{E h^{3}}{12\left(1-v^{2}\right)},
$$

and $E$ is Young's modulus;

$$
\begin{aligned}
& U_{\text {max }_{\text {comp osite }}}=\frac{1}{2} \int_{0}^{Y_{m m} \frac{(a-y)}{\tan \alpha}} \int_{\frac{y}{\tan \beta}}^{\frac{a}{\operatorname{an} \beta}}\left\{D_{11}\left(\frac{\partial \theta_{x}}{\partial x}\right)^{2}\right. \\
& +D_{22}\left(\frac{\partial \theta_{y}}{\partial y}\right)^{2}+2 D_{12}\left(\frac{\partial \theta_{x}}{\partial x} \frac{\partial \theta_{y}}{\partial y}\right) \\
& +D_{66}\left(\frac{\partial \theta_{x}}{\partial y}+\frac{\partial \theta_{y}}{\partial x}\right)^{2}+2 D_{16} \frac{\partial \theta_{x}}{\partial x}\left(\frac{\partial \theta_{x}}{\partial y}+\frac{\partial \theta_{y}}{\partial x}\right) \\
& +2 D_{26} \frac{\partial \theta_{y}}{\partial y}\left(\frac{\partial \theta_{x}}{\partial y}+\frac{\partial \theta_{y}}{\partial x}\right)+A_{44}\left(\theta_{y}+\frac{\partial w}{\partial y}\right)^{2} \\
& +2 A_{45}\left(\theta_{y}+\frac{\partial w}{\partial y}\right)^{2}\left(\theta_{x}+\frac{\partial w}{\partial x}\right) \\
& \left.+A_{55}\left(\theta_{x}+\frac{\partial w}{\partial x}\right)^{2}\right\} d x d y
\end{aligned}
$$

where coefficients $D_{i j},(i, j=1,2,6)$, are the bending, twisting and bending-twisting coupling rigidities, which are given by:

$$
D_{i j}=\frac{1}{3} \sum_{k=1}^{N_{c}} Q_{i j}^{(k)}\left(z_{k+1}^{3}-z_{k}^{3}\right),
$$

where $A_{i j},(i, j=4,5)$, are shear rigidity coefficients, given by:

$$
A_{i j}=k \sum_{k=1}^{N_{c}} Q_{i j}^{(k)}\left(z_{k+1}-z_{k}\right)
$$

where $k$ is the shear correction factor; coordinates $z_{k+1}$, and $z_{k}$ are depicted in Figure 2, $N_{c}$ is the total number of layers in the laminate, and $Q_{i j}$ are reduced transformed rigidities (see, for instance, [5]), which depend on the mechanical properties of each lamina and the angle of fiber orientation.

\subsection{Geometric mapping}

For generality and convenience, the present formulation is expressed in a dimensionless form. The actual trapezoidal plate in the $x-y$ physical domain is mapped into a square plate in the computational $u, v$ domain $(-1 \leq u \leq 1,-1 \leq v \leq 1)$ using the following coordinate transformation (see, for instance [7]):

$$
\begin{aligned}
& x=\sum_{i=1}^{4} N_{i}(u, v) x_{i}, \\
& y=\sum_{i=1}^{4} N_{i}(u, v) y_{i},
\end{aligned}
$$

where:

$$
\begin{aligned}
& \left(x_{1}, y_{1}\right)=(0,0), \\
& \left(x_{2}, y_{2}\right)=(a, 0), \\
& \left(x_{3}, y_{3}\right)=(a-c \cos \beta, c \sin \beta), \\
& \left(x_{4}, y_{4}\right)=(b \cos \alpha, b \sin \alpha) .
\end{aligned}
$$

The mapping functions, $N_{i}$, are defined by:

$$
N_{i}(u, v)=\frac{1}{4}\left(1+u_{i} u\right)\left(1+v_{i} v\right), \quad i=1,2,3,4,
$$

in which:

$$
\begin{array}{ll}
\left(u_{1}, v_{1}\right)=(-1,-1), & \left(u_{2}, v_{2}\right)=(1,-1), \\
\left(u_{3}, v_{3}\right)=(1,1), & \left(u_{4}, v_{4}\right)=(-1,1) .
\end{array}
$$

The derivatives of any quantity in the two coordinate systems are related by:

$$
\begin{aligned}
& \frac{\partial}{\partial x}=\Gamma_{11} \frac{\partial}{\partial u}+\Gamma_{12} \frac{\partial}{\partial v}, \\
& \frac{\partial}{\partial y}=\Gamma_{21} \frac{\partial}{\partial u}+\Gamma_{22} \frac{\partial}{\partial v},
\end{aligned}
$$

where $\Gamma_{i j}$ are the elements of $\mathbf{J}^{-1}$ in which:

$$
\mathbf{J}=\sum_{i=1}^{4}\left[\begin{array}{c}
\frac{\partial N_{i}}{\partial u} \\
\frac{\partial N_{i}}{\partial v}
\end{array}\right]\left[\begin{array}{ll}
x_{i} & y_{i}
\end{array}\right],
$$

is the Jacobian matrix of the transformation.

The infinitesimal area, $d A=|J| d u d v$, in the physical domain, is mapped in the computational domain according to:

$$
d A=|\mathbf{J}| d u d v,
$$

where $|\mathbf{J}|$ is the determinant of the Jacobian matrix.

The kinetic and strain energies of the vibration hybrid plate can now be expressed in mapping coordinates, as follows: 


$$
\begin{aligned}
& T_{\max _{\text {met }}}=\frac{1}{2} \int_{-1}^{1} \int_{-1}^{v_{m c}}\left\{\rho_{m} h\left(\frac{\partial w}{\partial t}\right)^{2}\right. \\
& +\frac{\rho_{m} h^{3}}{12}\left(\left(\Gamma_{11}^{2}+\Gamma_{12}^{2}\right)\left(\frac{\partial \theta_{u}}{\partial t}\right)^{2}+\left(\Gamma_{21}^{2}+\Gamma_{22}^{2}\right)\left(\frac{\partial \theta_{v}}{\partial t}\right)^{2}\right) \\
& \left.\left.+\left(2 \Gamma_{11} \Gamma_{12}+2 \Gamma_{21} \Gamma_{22}\right) \frac{\partial \theta_{u}}{\partial t} \frac{\partial \theta_{v}}{\partial t}\right)\right\} d v d u
\end{aligned}
$$$$
T_{\max _{\text {comp osite }}}=\frac{1}{2} \int_{-1}^{1} \int_{v_{m c}}^{1}\left\{m_{c}\left(\frac{\partial w}{\partial t}\right)^{2}\right.
$$$$
+I_{c}\left(\left(\Gamma_{11}^{2}+\Gamma_{12}^{2}\right)\left(\frac{\partial \theta_{u}}{\partial t}\right)^{2}+\left(\Gamma_{21}^{2}+\Gamma_{22}^{2}\right)\left(\frac{\partial \theta_{v}}{\partial t}\right)^{2}\right.
$$$$
\left.\left.+\left(2 \Gamma_{11} \Gamma_{12}+2 \Gamma_{21} \Gamma_{22}\right) \frac{\partial \theta_{u}}{\partial t} \frac{\partial \theta_{v}}{\partial t}\right)\right\} d v d u
$$$$
U_{\max _{\text {metal }}}=\frac{1}{2} \int_{-1}^{1} \int_{-1}^{v_{m c}}\{f(u, v)\} d v d u
$$$$
U_{\max _{\text {composite }}}=\frac{1}{2} \int_{-1}^{1} \int_{v_{m c}}^{1}\{g(u, v)\} d v d u
$$

where $f(u, v)$ and $g(u, v)$ are the functions that depend on the parameters of the problem, e.g. geometry, material properties and variables $u$ and $v$. Also, components of the displacement field in the $u, v$ and $z$ directions are given by:

$$
\begin{aligned}
& w(x, y)=w(u, v), \\
& \theta_{x}(x, y)=\Gamma_{11} \theta_{u}(u, v)+\Gamma_{12} \theta_{v}(u, v), \\
& \theta_{y}(x, y)=\Gamma_{21} \theta_{u}(u, v)+\Gamma_{22} \theta_{v}(u, v) .
\end{aligned}
$$

And $v_{m c}$ is the length of the boundary between the metal and thte composite in the mapping coordinates.

\subsection{Approximating functions}

The transvers deflection and the rotations are expressed by products of simple one-dimensional polynomials, as follows:

$$
\begin{aligned}
& w(u, v, t)=\sum_{i=1}^{M} \sum_{j=1}^{N} a_{i j}(t) U_{i}(u) V_{j}(v), \\
& \theta_{u}(u, v, t)=\sum_{i=1}^{M} \sum_{j=1}^{N} b_{i j}(t) \Theta_{i}(u) V_{j}(v),
\end{aligned}
$$

$$
\theta_{v}(u, v, t)=\sum_{i=1}^{M} \sum_{j=1}^{N} c_{i j}(t) U_{i}(u) \Phi_{j}(v)
$$

where $a_{i j}(t), b_{i j}(t)$ and $c_{i j}(t)$ are the unknown coefficients to be determined by the Ritz method. Also, $U_{i}(u), V_{j}(v), \Theta_{i}(u)$ and $\Phi_{j}(v)$ are polynomials that satisfy the geometry boundary conditions of the equivalent beam in each coordinate. It is well known that it is not necessary to subject coordinate functions to natural boundary conditions. It is sufficient that they satisfy geometrical boundary conditions since, as the number of coordinate functions approaches infinity, the natural boundary conditions will be exactly satisfied [1820]. Consequently, when the edges have rotational or translational restraints, all boundary conditions are natural. Thus, it is possible to ignore the boundary conditions in the construction of the first polynomial of each set.

Polynomials for boundary conditions c-f-f-f, s-s-s$\mathrm{s}$ and $\mathrm{c}-\mathrm{c}-\mathrm{c}-\mathrm{c}$ are given in the following:

c-f-f-f:

$$
\begin{aligned}
U_{i}(u) & =\frac{1}{2^{i} i !} \frac{d^{i}}{d u^{i}}\left(u^{2}-1\right)^{i}, & i & =1,2,3, \cdots \\
V_{j}(v) & =(v+1)^{j+1}, & j & =1,2,3, \cdots \\
\Theta_{i}(u) & =\frac{d U_{i}(u)}{d u}, & i & =0,1,2, \cdots \\
\Phi_{j}(v) & =\frac{d V_{i}(v)}{d v}, & j & =0,1,2, \cdots
\end{aligned}
$$

S-S-s-s:

$$
\begin{array}{ll}
U_{i}(u)=\sin (i \pi(u+1) / 2), & i=1,2,3, \cdots \\
V_{j}(v)=\sin (j \pi(v+1) / 2), & j=1,2,3, \cdots \\
\Theta_{i}(u)=\frac{d U_{i}(u)}{d u}, & i=0,1,2, \cdots \\
\Phi_{j}(v)=\frac{d V_{i}(v)}{d v}, & j=0,1,2, \cdots
\end{array}
$$

c-c-c-c:

$$
\begin{array}{ll}
U_{i}(u)=(u+1)(u-1) u^{(i-1)}, & i=1,2,3, \cdots \\
V_{j}(v)=(v+1)(v-1) v^{(j-1)}, & j=1,2,3, \cdots \\
\Theta_{i}(u)=\frac{d U_{i}(u)}{d u}, & i=0,1,2, \cdots \\
\Phi_{j}(v)=\frac{d V_{i}(v)}{d v}, & j=0,1,2, \cdots
\end{array}
$$




\section{Application of Ritz method}

Application of the Ritz method requires minimization of the following energy functional:

$$
\pi=U_{\max }-T_{\max }
$$

where $U_{\max }$ and $T_{\max }$ are, respectively, given by Eqs. (18)-(23). Minimization of the functional leads to the following system of equations:

$$
\left([\mathbf{k}]-w^{2}[\mathbf{M}]\right)\{\overline{\mathbf{c}}\}=\{\mathbf{0}\},
$$

where:

$$
\begin{aligned}
& \{\overline{\mathbf{c}}\}=\left\{\begin{array}{l}
a_{i j} \\
b_{i j} \\
c_{i j}
\end{array}\right\}, \\
& {[\mathbf{k}]=\left[\begin{array}{rrr}
{\left[\mathbf{k}^{w w}\right]} & {\left[\mathbf{k}^{w \theta_{x}}\right]} & {\left[\mathbf{k}^{w \theta_{y}}\right]} \\
& {\left[\mathbf{k}^{\theta_{x} \theta_{x}}\right]} & {\left[\mathbf{k}^{\theta_{x} \theta_{y}}\right]} \\
\operatorname{sym} & & {\left[\mathbf{k}^{\theta_{y} \theta_{y}}\right]}
\end{array}\right],} \\
& {[\mathbf{M}]=\left[\begin{array}{rrr}
{\left[\mathbf{M}^{w w}\right]} & {[\mathbf{0}]} & {[\mathbf{0}]} \\
& {\left[\mathbf{M}^{\theta_{x} \theta_{x}}\right]} & {[\mathbf{0}]} \\
\operatorname{sym} & & {\left[\mathbf{M}^{\theta_{y} \theta_{y}}\right]}
\end{array}\right] .}
\end{aligned}
$$

\section{Numerical results}

Numerical results, which are useful for appreciating the variations of frequency parameters for plates with different metal to composite ratios, different geometric conditions and different fiber orientation angles, are presented in this section. These results are obtained for two cases, including cantilevered plate simply supported at all sides of the plate.

\subsection{Convergence analysis}

It is well known that the Ritz method can lead to arbitrarily accurate approximate solutions to boundary value problems. Provided that the trial functions are admissible in the original variational principle, convergence to the true solution is guaranteed as the number of admissible functions tends to infinity [13]. In cases of dynamic problems, displacement-based Ritz methods show a monotonic convergence of the frequency solutions from above, i.e. upper bound values for the frequency parameters are obtained. In practical computations, the number of Ritz terms is limited by CPU time and capacity, and the achievable accuracy of the solution is influenced by this truncation. Moreover, the choice of trial functions strongly affects the rate of convergence of the method and, thus, its efficiency. This aspect is also related to the numerical stability of the solution, since ill conditioning may occur when many admissible functions are required to obtain a desired accuracy. Considering this, the evaluation of any Ritz-based approach should first include an appropriate convergence analysis. The convergence study of the proposed Ritz formulation is presented in this section. Convergence for the first six values of the neutral frequency for boron-epoxy $[ \pm 45]_{2 s}$ and aluminum for hybrid trapezoidal plates with $(v=0.3$, $k=0.833, \alpha=\beta=10^{\circ}$ ) under two boundary conditions, s-s-s-s and c-f-f-f, are shown in Table 1.

\subsection{Verification of the method}

To validate the proposed approach, comparisons with numerical values provided by other researchers ob-

Table 1. Convergence study of the first six values of the neutral frequency for boron-epoxy $[ \pm 45]_{2 s}$ and aluminum for hybrid trapezoidal plates $\left(v=0.3, k=0.833, \alpha=\beta=10^{\circ}\right)$.

\begin{tabular}{ccccccc}
\hline $\boldsymbol{M}=\boldsymbol{N}$ & $\boldsymbol{\omega}_{\mathbf{1}}$ & $\boldsymbol{\omega}_{\mathbf{2}}$ & $\boldsymbol{\omega}_{\mathbf{3}}$ & $\boldsymbol{\omega}_{\mathbf{4}}$ & $\boldsymbol{\omega}_{\mathbf{5}}$ & $\boldsymbol{\omega}_{\mathbf{6}}$ \\
\hline 2 & & & $\mathbf{s}-\mathbf{s}-\mathbf{s}-\mathbf{s}$ & & & \\
3 & 245.67 & 606.89 & 686.90 & 1160.84 & 1378.9 & 1554.3 \\
4 & 228.65 & 485.67 & 538.78 & 897.33 & 1127.4 & 1300.1 \\
5 & 227.33 & 484.58 & 537.66 & 852.54 & 1113.6 & 1255.5 \\
6 & 227.21 & 484.40 & 537.42 & 849.39 & 1108.5 & 1240.7 \\
7 & 227.10 & 484.32 & 537.01 & 848.18 & 1105.7 & 1236.9 \\
\hline & & & $\mathbf{c - f - f - f}$ & & & \\
\hline 2 & 36.78 & 140.41 & 381.24 & 719.40 & 815.45 & 1037.45 \\
3 & 30.82 & 116.75 & 168.43 & 369.46 & 398.74 & 483.25 \\
4 & 29.14 & 102.68 & 147.44 & 335.55 & 352.22 & 422.19 \\
5 & 28.51 & 93.89 & 132.65 & 298.23 & 330.18 & 390.18 \\
6 & 28.32 & 91.31 & 128.95 & 285.34 & 318.39 & 361.39 \\
7 & 28.29 & 91.14 & 128.68 & 283.21 & 314.03 & 355.47 \\
\hline
\end{tabular}


tained by other methods, are carried out. Also, convergence studies have been implemented.

Results of a convergence study of the frequency parameter, $\omega$, are shown in Table 1 . The first four values of $\omega$ are presented for trapezoidal thick plates $\left(\alpha=\beta=20^{\circ}\right)$, cantilever, simply supported at the sides. The plate consists of a single boron-epoxy layer $\left(\theta=45^{\circ}\right)$ with $E_{1}=207 \mathrm{GPa}, E_{2}=21 \mathrm{GPa}, v_{12}=0.3$, $G_{12}=G_{13}=7 \mathrm{GPa}, G_{23}=4.2 \mathrm{GPa}, k=5 / 6$, and aluminum with the mechanical properties $E=73 \mathrm{GPa}$, $v=0.3, \rho=2800 \mathrm{~kg} / \mathrm{m}^{3}$, span $=2 \mathrm{~m}, a=2 \mathrm{~m}$ and $k=5 / 6$.

The convergence of the mentioned frequency parameters is studied by gradually increasing the number of polynomials in the approximate function, $w, \Theta_{u}$ and $\Theta_{v}$, which are, respectively, given by $M$ and $N$. It can be observed that the frequency parameters converge monotonically from above as the number of terms increases.

Table 2 shows the values of the fundamental frequency parameter, $\Omega=\omega \operatorname{span}^{2} \sqrt{\rho} / D / 2 \pi$, for different isotropic trapezoidal plates, simply supported at the four sides. On the other hand, frequency parameters are compared with those of Haldar and Manna [16] who employed high precision triangular elements including shear strain. They are also compared with the results of Zhong [12] who used the DSC method and with the results of Quintana et al. [21] who worked on the Ritz method. Zhong [12] also presents a convergence study increasing the number of grid points. The numerical values obtained by these authors are depicted in Tables 2 and 3 and have been computed using $15 \times 15$ grid points. It is important to point out that the values of the frequency parameters, obtained by the methodology proposed in this work, are obtained using seven terms in the coordinate functions in each direction $(M=N=7)$.

It should be noted that the Ritz method produces approximations from above for each eigenvalue with respect to the exact eigenvalues. It is important when the exact solution cannot be obtained. In Table 3, each layer of the lamina has the following material properties:

$$
\begin{aligned}
& E_{1}=40 \mathrm{GPa}, \\
& E_{2}=0.6 \mathrm{GPa}, \\
& v_{12}=0.25, \\
& G_{12}=G_{13}=0.6 E_{2}, \\
& G_{23}=0.5 E_{2} \\
& k=0.833 .
\end{aligned}
$$

\begin{tabular}{|c|c|c|c|c|c|c|c|c|c|c|}
\hline \multirow[b]{2}{*}{$\tan \alpha$} & \multirow[b]{2}{*}{$1-\operatorname{span}$} & \multirow[b]{2}{*}{$h / \operatorname{span}$} & \multicolumn{4}{|c|}{ SSSS } & \multicolumn{4}{|c|}{$\mathrm{CCCC}$} \\
\hline & & & Present & $\begin{array}{l}\text { Ref. } \\
{[18]}\end{array}$ & $\begin{array}{l}\text { Ref. } \\
{[12]}\end{array}$ & $\begin{array}{l}\text { Ref. } \\
{[11]}\end{array}$ & Present & $\begin{array}{l}\text { Ref. } \\
{[\mathbf{1 8}]}\end{array}$ & $\begin{array}{l}\text { Ref. } \\
{[12]}\end{array}$ & $\begin{array}{r}\text { Ref. } \\
{[11]}\end{array}$ \\
\hline 0.4 & 0.8 & 0.1 & 27.509 & 27.50 & 27.54 & 34.74 & 34.767 & 34.76 & 34.76 & 34.74 \\
\hline 0.3 & 0.6 & & 23.836 & 23.82 & 24.06 & 23.91 & 30.498 & 30.39 & 31.08 & 30.95 \\
\hline 0.2 & 0.4 & & 20.331 & 20.32 & 20.48 & 20.35 & 27.543 & 27.53 & 27.62 & 27.53 \\
\hline 0.1 & 0.2 & & 17.760 & 17.37 & 18.41 & 17.39 & 24.903 & 24.75 & 25.12 & 24.73 \\
\hline 0.4 & 0.8 & 0.2 & 17.538 & 17.53 & 17.63 & 17.54 & 19.568 & 19.47 & 19.51 & 19.54 \\
\hline 0.3 & 0.6 & & 15.455 & 15.39 & 15.46 & 15.44 & 17.486 & 17.47 & 17.56 & 17.45 \\
\hline 0.2 & 0.4 & & 13.487 & 13.48 & 13.51 & 13.49 & 15.801 & 15.79 & 15.88 & 15.76 \\
\hline 0.1 & 0.2 & & 11.975 & 11.97 & 11.99 & 11.97 & 14.557 & 14.50 & 14.59 & 14.46 \\
\hline
\end{tabular}

Table 2. Dimensionless parameters of fundamental frequency, $\Omega=\omega \operatorname{span}^{2} \sqrt{\rho h} / D / 2 \pi$, for simply supported isotropic trapezoidal plates $(v=0.3, k=0.833, \alpha=\beta)$.

\begin{tabular}{ccccccc}
\hline $\tan \boldsymbol{\alpha}$ & $\mathbf{1}-$ span & $\boldsymbol{h} / \boldsymbol{b}$ & Present & $\begin{array}{r}\text { Ref. } \\
{[\mathbf{1 8}]}\end{array}$ & $\begin{array}{c}\text { Ref. } \\
{[\mathbf{8}]}\end{array}$ & $\begin{array}{c}\text { Ref. } \\
{[\mathbf{7}]}\end{array}$ \\
\hline 0.4 & 0.8 & 0.01 & 6.013 & 6.02 & 5.99 & 6.02 \\
0.3 & 0.6 & & 4.903 & 4.91 & 4.90 & 4.91 \\
0.2 & 0.4 & & 4.066 & 4.08 & 4.06 & 4.09 \\
0.1 & 0.2 & & 3.522 & 3.51 & 3.51 & 3.52 \\
\hline
\end{tabular}

Table 3. Dimensionless parameters of fundamental frequency, $\Omega=\omega \operatorname{span}^{2} \sqrt{\frac{\rho / E_{2}}{h}}$, for cross ply $[90 / 0 / 0 / 90]$ trapezoidal plate $(\alpha=\beta)$. 
Table 4. Frequency parameters, $\omega$, for cantilevered skewed trapezoidal plates $\left(\beta=45^{\circ}\right)$.

\begin{tabular}{cccccc}
\hline $\boldsymbol{c} / \boldsymbol{a}$ & $\boldsymbol{d} / \boldsymbol{a}$ & $\boldsymbol{\omega}_{\mathbf{1}}$ & $\boldsymbol{\omega}_{\mathbf{2}}$ & $\boldsymbol{\omega}_{\mathbf{3}}$ & $\boldsymbol{\omega}_{\mathbf{4}}$ \\
\hline \multirow{4}{*}{0.5} & 1 & 707.36 & 1281.58 & 2761.09 & 5584.47 \\
& 0.5 & 792.10 & 1692.02 & 4666.14 & 6124.4 \\
& 0.25 & 1076.65 & 3012.97 & 5345.75 & 7890.07 \\
& 1 & 164.02 & 436.83 & 1083.52 & 1464.93 \\
1 & 0.5 & 184.42 & 646.98 & 1118.86 & 1841.37 \\
& 0.25 & 229.28 & 956.38 & 1471.36 & 2573.35 \\
& & & & & \\
2 & 1 & 38.04 & 151.90 & 335.25 & 467.76 \\
& 0.5 & 44.86 & 194.16 & 357.44 & 601.88 \\
& 0.25 & 51.66 & 211.06 & 389.02 & 673.47 \\
\hline
\end{tabular}

Results for two different boundary conditions, (ssss and $\mathrm{cccc})$, thickness ratios $(h / \mathrm{span}=0.1,0.2)$, and several geometric configurations have been included in this table. In all cases, a very good agreement in the numerical values can be observed, indicating the accuracy that can be achieved through application of this methodology, which uses simple polynomials to construct the shape functions.

\subsection{New numerical results}

In this section, new numerical results, which can serve as a supplement to the existing data, based on the vibration characteristics of moderately thick trapezoidal hybrid plates, are presented. In particular, the results for the different geometric conditions of the plate are presented.

Table 4 shows the frequency parameters obtained by the present method for the first four modes of the 9 skewed plate configurations, having $\beta=45^{\circ}$ and a wide range of aspect ratios $(c / a=0.5,1,2)$, metal to composite ratio $\left(Y_{m c}=0.5\right)$, and chord ratio $(d / a=0.25,0.5,1)$, as shown in Figure 2. Table 5 shows frequencies obtained for the first four modes where results have been calculated for $\beta=0^{\circ}$ to $-60^{\circ}$.

To evaluate the effect of different fiber orientation angles, $\theta$, on the dynamic properties of trapezoidal laminated plates $\left(\alpha=\beta=20^{\circ}\right)$, variation of the frequency parameter for the first four modes of the hybrid plate is plotted in Figure 3. The elastic properties and geometrical parameters used in Figure 3 are as the data shown in Table 1 , but with $\operatorname{span}=1 \mathrm{~m}$ and $a=1.2 \mathrm{~m}$.

The effect of the metal to composite ratio is shown in Figure 4. Variation of the frequency parameter for the first four modes of the hybrid plate is plotted in Figure 4 . The data of Figures 3 and 4 are the same, but with different fibre angles and metal to composite ratios. To examine the effects of the metal to composite ratio and different angles, $\alpha$, the frequency
Table 5. Frequency parameters, $\omega$, for cantilevered skewed trapezoidal plates $(a / b=1)$.

\begin{tabular}{cccccc}
\hline $\begin{array}{c}\text { Skew } \\
\text { angle } \\
(\boldsymbol{\beta})\end{array}$ & $\boldsymbol{d} / \boldsymbol{a}$ & $\boldsymbol{\omega}_{\mathbf{1}}$ & $\boldsymbol{\omega}_{\mathbf{2}}$ & $\boldsymbol{\omega}_{\mathbf{3}}$ & $\boldsymbol{\omega}_{\mathbf{4}}$ \\
\hline $0^{\circ}$ & 1 & 132.01 & 359.84 & 593.76 & 1000.41 \\
& 0.5 & 149.55 & 450.81 & 636.53 & 1391.11 \\
& 0.25 & 174.79 & 492.81 & 717.21 & 1697.60 \\
& 1 & 130.99 & 367.68 & 642.01 & 975.80 \\
$15^{\circ}$ & 0.5 & 147.59 & 423.21 & 683.96 & 1311.72 \\
& 0.25 & 181.34 & 460.44 & 750.14 & 915.23 \\
& 1 & 143.84 & 394.20 & 815.33 & 1090.88 \\
$30^{\circ}$ & 0.5 & 157.85 & 446.82 & 837.43 & 1471.68 \\
& 0.25 & 182.26 & 603.05 & 875.15 & 1945.45 \\
& 1 & 132.94 & 408.40 & 1121.22 & 1222.29 \\
$45^{\circ}$ & 0.5 & 139.72 & 553.28 & 1060.13 & 1758.16 \\
& 0.25 & 166.91 & 832.80 & 1322.37 & 2287.21 \\
& 1 & 197.81 & 650.26 & 1922.85 & 2231.92 \\
$60^{\circ}$ & 0.5 & 200 & 836.58 & 2107.13 & 2921.69 \\
& 0.25 & 254.99 & 1184.81 & 2469.12 & 3562.45 \\
\hline
\end{tabular}

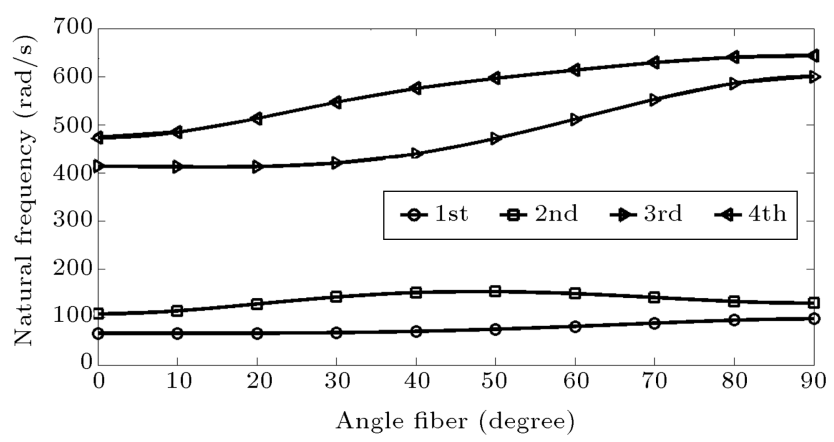

Figure 3. Effect of fiber orientation on the frequency parameter for first four modes, for a four-layer $[0 / \theta / \theta / 0]$ laminated plate.

parameter for the first four modes is considered. The dimensions and properties used in Figure 5. are listed in Figure 4.

Figure 6 shows the variation of weight to thickness of the plate, with variations of $Y_{m c}$.

\section{Conclusion}

A simple, accurate and general algorithm for the free transverse vibration analysis of trapezoidal hybrid plates, consisting of symmetrically laminated composite in combination with an isotropic metal, is proposed in this study. The developed methodology is based on the global Ritz method and the first order shear deformation theory. The transverse deflection and 


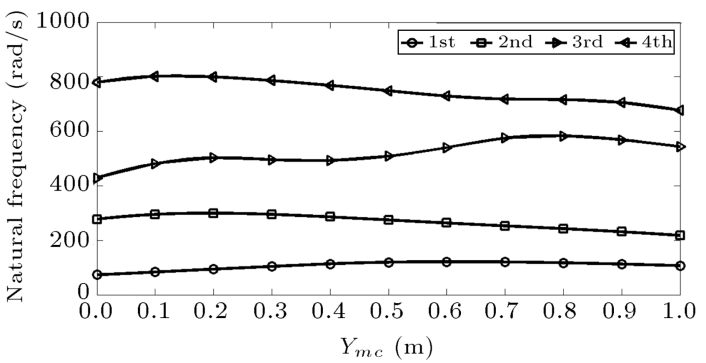

(a) $[-30 /+30 /+30 /-30]$

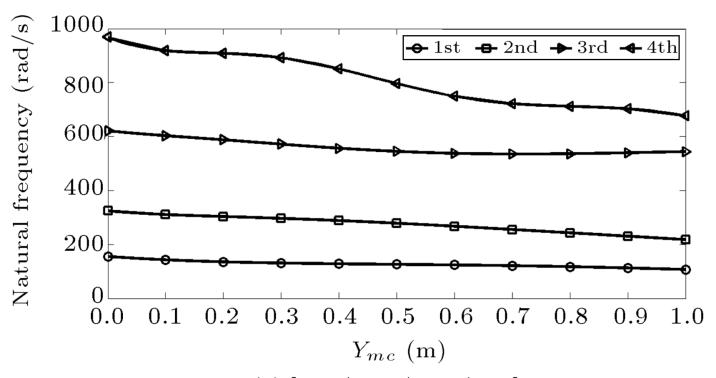

(c) $[-60 /+60 /+60 /-60]$

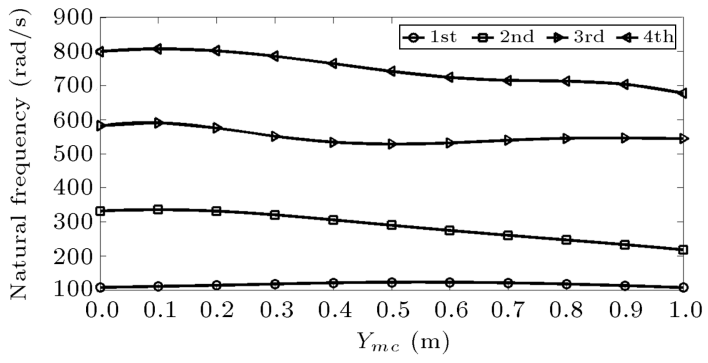

(b) $[-45 /+450 /+45 /-45]$

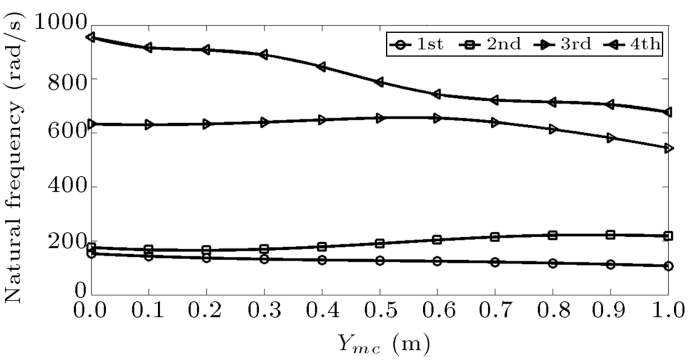

(d) $[0 / 90 / 90 / 0]$

Figure 4. Variation of frequency parameters, $\omega$, with metal to composite ratio, $Y_{m c}$, in different angels fiber.

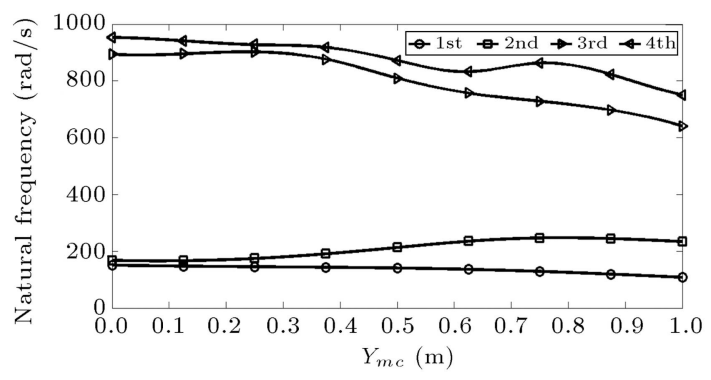

(a) $\left(\alpha=0^{\circ}, \beta=0^{\circ}\right)$

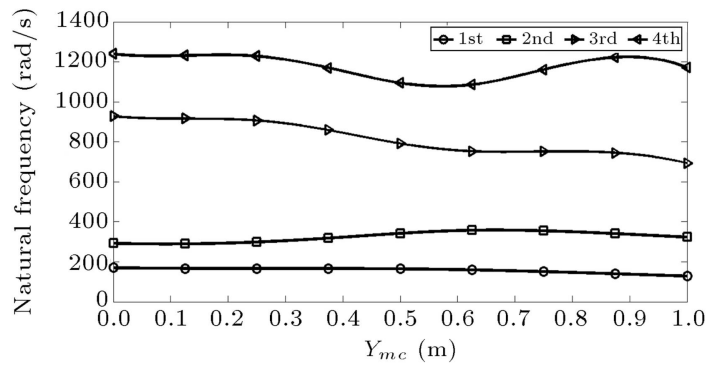

(c) $\left(\alpha=30^{\circ}, \beta=0^{\circ}\right)$

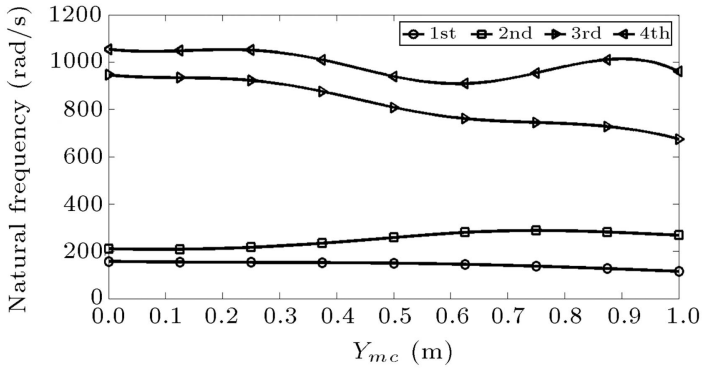

(b) $\left(\alpha=15^{\circ}, \beta=0^{\circ}\right)$

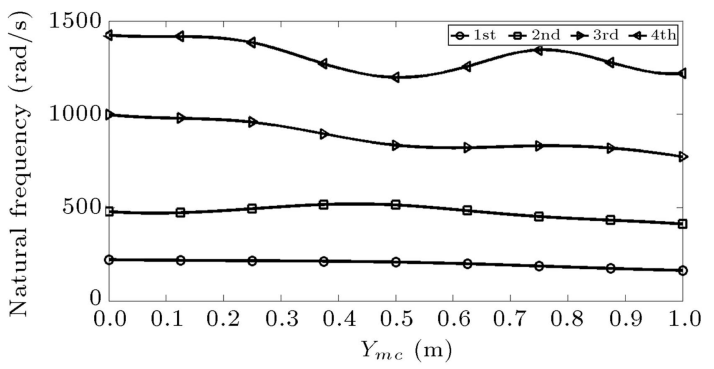

(d) $\left(\alpha=45^{\circ}, \beta=0^{\circ}\right)$

Figure 5. Variation of frequency parameters, $\omega$, with metal to composite ratio, $Y_{m c}$, in $\alpha=0^{\circ}, 15^{\circ}, 30^{\circ}, 45^{\circ}$, and $\beta=0^{\circ}$.

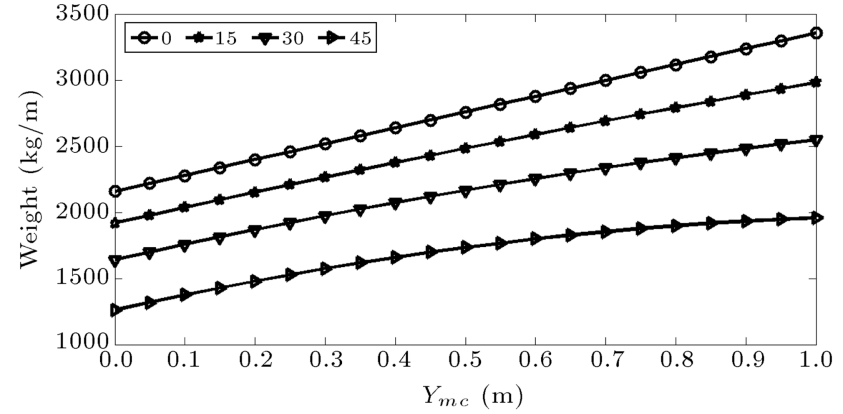

Figure 6. Effect of metal to composite ratio on variation of weight to thickness of plate. two rotations are approximated by means of simple polynomials. The algorithm allows unified treatment of hybrid plates with different geometrics, aspect ratios, number of layers, staking sequences and metal to composite ratios. From convergence studies and comparisons with results available in the literature, it is observed that the approach presented is reliable and accurate. Sets of numerical results are given in tabular and graphical form, illustrating the influence of different geometrics, staking sequences and metal to composite ratios. Finally, it is important to note that the proposed method can be easily extended for 
application of static and stability analyses. It can also be generalized to study thick trapezoidal plates with a non-symmetrical stacking sequence in the midplane.

\section{References}

1. Kapania, R.K. and Liu, Y. "Static and vibration analyses of general wing structures using equivalent plate models", AIAA Journal, 38(7), pp. 1269-1277 (July 2000).

2. Livne, E. "Equivalent plate structural modeling for wing shape optimazation including transverse shear", AIA A Journal, 32(6), pp. 1278-1288 (June 1994).

3. Vepa, R. "Aeroelastic analysis of wing structures using equivalent plate models", AIA A Journal, 46(5), pp. 1216-1225 (May 2008).

4. Kapidzic, Z. "Strength analysis and modeling of hybrid composite-aluminum aircraft structures", Linkoping Studies in Science and Technology, Licentiate Thesis No. 1590 (May 2013).

5. Reddy, J.N., Mechanics of Laminated Anisotropic Plates: Theory and Analysis, Boca Raton, FL: CRC Press (1997).

6. Whitehead, R.S. "Lessons learned for composite structures", The First NASA Advanced Composites Technology Conference, Part I, 399-415, Seattle (1990).

7. Kitipornchni, S., Xiang, Y., Liew, K.M. and Lim, M.K. "A global approach for vibration of thick trapezoidal plate", Computers and Structures, 53, pp. 83-92 (1994).

8. Liew, K.M., Xiang, Y. and Kitipornachi, S. "Research on thick plate vibration: A literature survey", Journal of Sound and Vibration, 180, pp. 163-176 (1995).

9. Karunasena, W., Kitipornchai, S., Al-Bermani, F.G.A. "Free vibration of cantilevered arbitrary triangular mindlinplate", Int. J. MechSci., 38, pp. 31-42 (1996).

10. Karunasena, W. and Kitipornchai, S. "Free vibration of shear-deformable general triangular plates", Journal of Sound and Vibration, 199, pp. 595-613 (1997).

11. Wu, L. and Liu, J. "Free vibration analysis of arbitrary shaped thick plates by differential cubature method", International Journal of Mechanical Sciences, 47, pp. 63-81 (2005).

12. Zhong, H.Z. "Free vibration analysis of isosceles triangular mindlin plate by the triangular differential quadrature method", Journal of Sound and Vibration, 237, pp. 697-708 (2000).

13. Dozio, L. and Carrera, E. "A variable kinematic Ritz formulation for vibration study of quadrilateral plates with arbitrary thickness", Journal of Sound and Vibration, 330, pp. 11-32 (2011).

14. Kapania, R.K. and Lovejoy, A.E. "Free vibration of thick generally laminated cantilever quadrilateral plates", American Institute of Aeronautics and Astronautics, 34, pp. 74-86 (1996).

15. Chen, C.C., Kitipornachai, S., Lim, C.W. and Liew, K.M. "Free vibration of cantilevered symmetrically laminated thick trapezoidal plates", International Journal of Mechanical Sciences, 41, pp. 685-702 (1999).

16. Haldar, S. and Manna, M.C. "A high precision shear deformable element for free vibration of thick/thin composite trapezoidal plates steel", Composite Structures, 3, pp. 213-219 (2003).

17. Gurses, M., Civalek, O., Ersoy, H. and Kiracioglu, O. "Analysis of shear deformable laminated composite trapezoidal plates", Materials and Design, 30, pp. 3030-3035 (2009).

18. S.M. Dickinson, A. and DiBlasio, A. "On the use of orthogonal polynomials in the Rayleigh-Ritz method for the study of the vibration and buckling of isotropic and orthotropic rectangular plates", Journal of Sound and Vibration, 108, pp. 51-62 (1986).

19. Grossi, R. and Nallim, L. "Boundary and eigenvalue problem for generally restrained anisotropic plates", Proceedings of the Institution of Mechanical Engineers, Part K J. Multi-Body Dyn., 217, pp. 241-251 (2003).

20. Xiang, Y., Liew, K.M. and Kitipornchai, S. "Vibration analysis of rectangular Mindlin plates resting on elastic edge supports", Journal of Sound and Vibration, 204, pp. 1-16 (1997).

21. Quintana, M.V. and Nallim, L.G. "A general Ritz formulation for the free vibration analysis of the thick trapezoidal and triangular laminated plates resting on elastic supports", International Journal of Mechanical Sciences, 69, pp. 1-9 (2013).

22. Shokrollahi, S. and Bakhtiari-Nejad, F. "Limit-cycle oscillatios of swept-back trapezoidal wings at low subsonic flow", J. Aircraft, 41(4), American Institute of Aeronautics and Astronautics, pp. 948-953 (2004).

23. Zamani, M., Fallah, A. and Aghdam, M.M. "Free vibration analysis of moderately thick trapezoidal symmetrically laminated plates with various combinations of boundary conditions", European Journal of Mechanics A/Solids, 36, 204-212 (2012).

24. Civalek, O. "Free vibration analysis of symmetrically laminated composite plates with first-order shear deformation theory (FSDT) by discrete singular convolution method", Finite Elements in Analysis and Design, 44, pp. 725-731 (2008).

25. Wang, X. and Wu, Z. "Differential quadrature analysis of free vibration of rhombic plates with free edges", Applied Mathematics and Computation, 225, pp. 171183 (2013).

26. Civalek, O. "A four-node discrete singular convolution for geometric transformation and its application to numerical solution of vibration problem of arbitrary straight-sided quadrilateral plates", Applied Mathematical Modeling, 33, pp. 300-314 (2009). 


\section{Biographies}

Saeed Shokrollahi received his BS degree in Mechanical Engineering from Shiraz University, Iran, in 1994, his MS degree in Mechanical Engineering from Tehran University in 1997, and his $\mathrm{PhD}$ degree in Mechanical Engineering from Amirkabir University of Technology, Tehran, Iran, in 2004. He is currently Assistant Professor of Mechanical Engineering in the Aerospace Engineering Department of MalekAshtar University of Technology, Tehran, Iran. His research interests are in the field of aeroelasticity, including the study of dynamic interaction between aerodynamic flow and elastic structures, such as air- craft wings during high speed flight. He has also undertaken research in experimental modal analysis, nonlinear identification, structural dynamics, and unsteady aerodynamics. He has supervised more than thirty $\mathrm{Ms}$ theses and three $\mathrm{PhD}$ dissertations since 2004.

Salman Shafaghat received his BS degree in Mechanical Engineering from Sari Azad University, Iran, in 2010, and is currently studying for his MS degree in Aerospace Engineering at Malek-Ashtar University, Tehran, Iran. His degree thesis is regarding aeroelastic analysis of hybrid wings with low aspect ratio in supersonic flow. 